\title{
Phaeopterus unicolor A. Costa, 1856 (Coleoptera, Omalisidae): nuovi ritrovamenti italiani, note nomenclaturali e biologiche
}

\author{
Fabrizio Fanti ${ }^{1}$, Francesco Vitali ${ }^{2}$ \\ ${ }^{1}$ Corresponding author: Fabrizio Fanti, Via del Tamburino 69, I-53040 Piazze (SI), Italia. E-mail: fantifab@alice.it \\ 2 7a, rue Jean-Pierre Huberty, L-1742, Luxembourg. E-mail: vitalfranz@yahoo.de
}

\begin{abstract}
Riassunto - A seguito del recente ritrovamento di alcuni esemplari di Phaeopterus unicolor A. Costa, 1856, viene aggiornata la distribuzione italiana della specie e sono delineate alcune caratteristiche biologiche. La data di pubblicazione originaria viene inoltre anticipata di un anno, risultando la specie già validamente descritta nel Rendiconto della Società Reale Borbonica - Accademia delle Scienze di Napoli nel 1856.
\end{abstract}

Abstract - As a result of the recent discovery of a few specimens of Phaeopterus unicolor A. Costa, 1856 in continental Italy, the distribution of the species is updated and some biological characteristics are outlined. The publication date, currently reported as 1857 , has been found to be one year earlier: the species was indeed firstly described in the Rendiconto della Società Reale Borbonica - Accademia delle Scienze di Napoli in 1856.

Key-words: Coleoptera Omalisidae, Phaeopterus, taxonomy, faunistic, biology.

\section{INTRODUZIONE}

Phaeopterus unicolor fu descritto da Achille Costa (1856) su un esemplare maschio dei dintorni di Napoli. Il genere, monotipico, fu considerato per molto tempo sottogenere (Bertolini, 1872-1878; Heyden et alii, 1891, 1906; Luigioni, 1929; Porta, 1929; Kleine, 1942) o perfino sinonimo di Omalisus Geoffroy, 1762 (Bourgeois, 1882a, 1882b, 1884-1892; Bertolini, 1904; Champion, 1911). Benché già Reitter (1911) e Winkler (1989) avessero notato che i due generi erano da ritenersi separati, Phaeopterus è stato riconsiderato valido solo recentemente da Bocak \& Brlik (2008). Secondo tali autori, la specie è nota solo del complesso sardo-corso e, per alcune antiche segnalazioni, della zona costiera dell'Italia centro-meridionale, con un solo dato dell'interno (Leoni, 1907: Cerchio, Abruzzo).

Lo studio di due esemplari recentemente raccolti ci ha dato l'occasione per chiarire il lavoro in cui è apparsa la descrizione originale e per aggiornare la distribuzione geografica della specie evidenziandone alcune caratteristiche biologiche.

\section{MATERIALI E METODI}

Toscana: 1 No, Siena, Monte Cetona, loc. I Cancelli, $790 \mathrm{~m}, 42.55 \mathrm{~N}, 11.52 \mathrm{E}, 2$. VI.2012, da battitura dei rami bassi ("ombrellatura") di un giovane esemplare di Quercus cerris L. lungo un sentiero al margine del bosco, intorno alle 18:30, leg. e coll. Fanti.

LAZIO: 1 Oे, Roma, Cinecittà, sotto acquedotto romano, VI.1966, leg. e coll. F. Angelini.

\section{DISCUSSIONE}

Secondo molti autori (Bourgeois, 1882b, 1884-1892; Heyden et alii, 1906; Kleine, 1942; Bocak \& Brlik, 2008), Phaeopterus unicolor fu descritto da Achille Costa (1857) in una nota apparsa sulle Memorie della Reale Accademia delle Scienze di Napoli. Tuttavia la prima descrizione valida della specie è quella apparsa, seppur in forma più breve e senza disegno, nel Rendiconto della Società Reale Borbonica - Accademia delle Scienze (Costa, 1856). Una volta preparata la nota veniva, infatti, letta di fronte all'Accademia che ne pubblicava poi un sunto e, solo in seguito ad approvazione - nel caso specifico l'anno successivo - veniva integralmente edita (Anonimo, 1857).

Trattandosi di un genere monotipico anche la descrizione del genere va riferita alla medesima pubblicazione. Perciò la data di descrizione dei due taxa è la seguente:

Phaeopterus A. Costa, 1856 (Rendiconto della Società Reale Borbonica - Accademia delle Scienze 5 (Nuova Serie): 17.

Phaeopterus unicolor A. Costa, 1856 (Rendiconto della Società Reale Borbonica - Accademia delle Scienze 5 (Nuova Serie): 17.

La collezione Costa, considerata in genere distrutta (Bocak \& Brlik, 2008), è in realtà tuttora esistente, anche se difficilmente accessibile e in uno stato di conservazione presumibilmente cattivo (Pantaleoni, in litt.).

Phaeopterus unicolor è noto solo per pochissimi esemplari, raccolti perlopiù a cavallo tra XIX e XX secolo. Descritto dei dintorni di Napoli, fu citato per la prima volta in una lettera di Alexander Henry Haliday 
al collega Hermann Loew (Haliday, 1869). Il ditterologo racconta di averne catturato un esemplare a Lucca, nella primavera del 1867, rimarcandone l'importanza, giacché la specie era nota solo dell'Italia meridionale. In base ai riferimenti nel testo, la lettera è databile con certezza alla primavera del 1869 e si tratterebbe perciò del secondo reperto storico - ancora inedito - di questa specie, oltre che del più settentrionale noto sinora. L'insetto potrebbe essere, a tutt'oggi, conservato al National Museum of Ireland, dove la collezione pervenne, in parte distrutta, nel 1882.

Phaeopterus unicolor fu in seguito segnalato genericamente di Sardegna, Corsica, Lazio e Abruzzo (Baudi di Selve, 1872 [sub Homalisus spinosus n.n., coll. Gené];

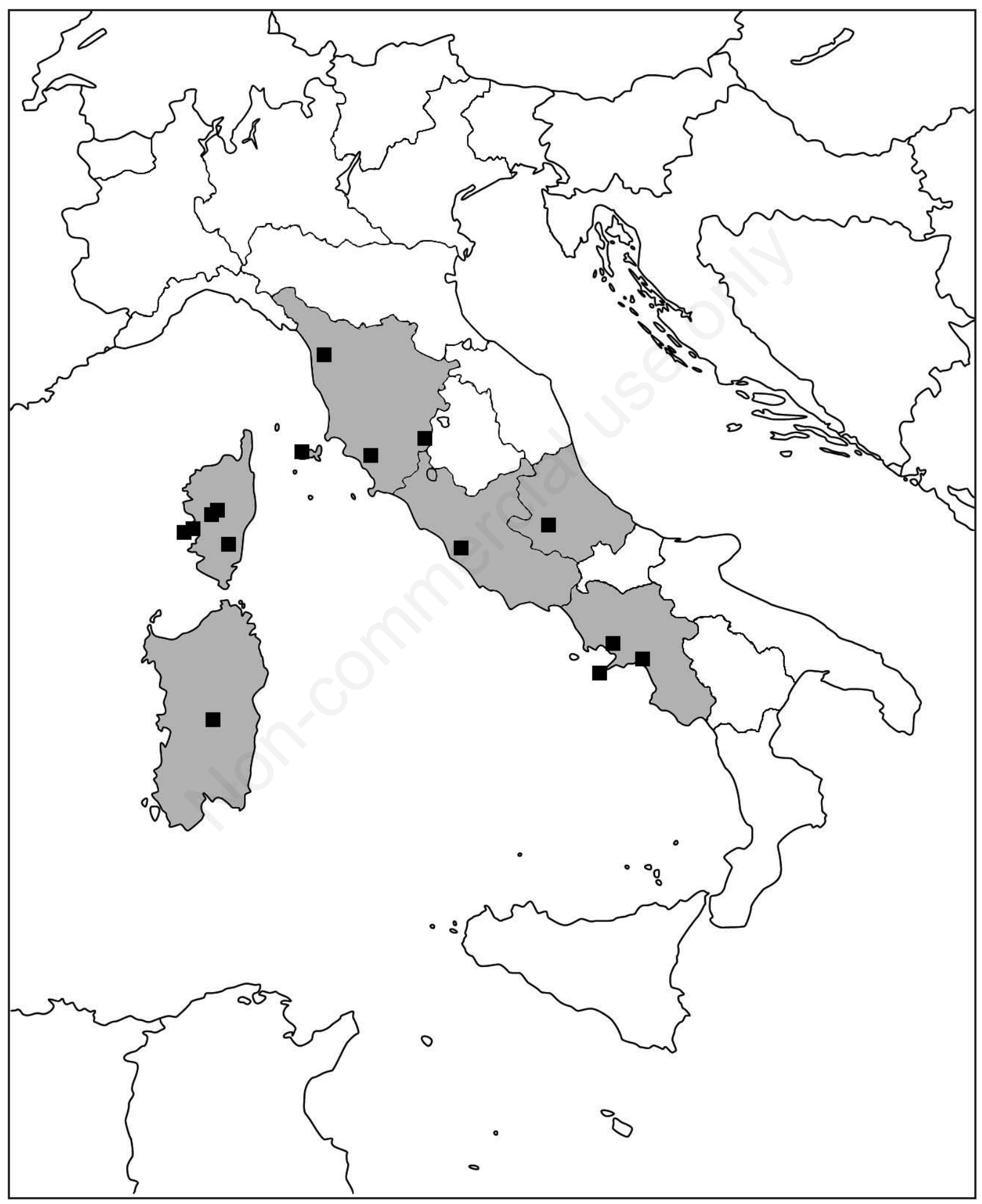

Fig. 1 - Distribuzione geografica nota di Phaeopterus unicolor A. Costa, 1856. / Known geographic distribution of Phaeopterus unicolor A. Costa, 1856. 
Bertolini, 1872-1878, 1904; Bargagli, 1873; Bourgeois, 1882a, 1882b, 1884-1892; Heyden et alii, 1891; SainteClaire Deville, 1914; Schaufuss, 1916; Luigioni, 1929; Porta, 1929; Kleine, 1942), ma con pochissime segnalazioni precise in territorio italiano: Roma (Heyden et alii, 1891, 1906; Sainte-Claire Deville, 1914; Schaufuss, 1916), Cerchio (Leoni, 1907), Aritzo (Champion, 1911) e Isola di Capri (Luigioni, 1929; Porta, 1929). Più precise le segnalazioni per la Corsica: Ajaccio, Bocognano, Forêt de Bavella, Bois du Salario presso Ajaccio, Ucciani (Bourgeois, 1882b; Sainte-Claire Deville, 1914, 1921, 1926; Bocak \& Brlik, 2008).

$\mathrm{Fu}$ inoltre segnalato da Bourgeois (1882a) di Collioure, nei Pirenei orientali francesi, dato poi ripreso successivamente da diversi Autori (Bourgeois, 1882b, 1884-1892; Heyden et alii, 1891; Sainte-Claire Deville, 1914; Schaufuss, 1916; Hicker, 1925), probabilmente anche come "Gallia meridionalis" (Hicker, 1925): tuttavia in questo caso ci sembra lecito ipotizzare una errata identificazione con Thilmanus laticeps Pic, 1913.

Pic (1938) lo ridescrisse come Homalisus minutus su un esemplare di origine italiana appartenente alla propria collezione e Bocak \& Brlik (2008) ci informano che l'olotipo, conservato oggi al Museo di Parigi, fu catturato a Poggio Cavallo (Grosseto) nell'aprile del 1899. L'esemplare senza località in collezione Grenier non è invece un paratipo, come sostenuto da tali autori, in quanto non citato nella descrizione originale.

Oltre a chiarire la tassonomia della specie, Bocak \& Brlik hanno anche aggiunto dati più precisi e nuove località, soprattutto di esemplari antichi. Per l'Italia: Salerno dint., 15-21.V.1903, Fratte leg. (Museo di Parigi); Roma, coll. C. Strasser (Senckenberg Deutsches Entomologisches Institut, Müncheberg), e una sola segnalazione recente: Isola d'Elba, M. Perone, 600-700 m, 28.IV.2007, coll. M. Geiser.

I recenti ritrovamenti - dopo vari decenni dalle ultime segnalazioni - ci permettono di confermare che la specie è presente, seppure rarissima, in Italia continentale (Fig. 1). In particolare, il reperto toscano è, non solo l'unico noto per la Toscana continentale dopo gli esemplari ottocenteschi di Haliday e Pic, ma anche il secondo ritrovamento in una località italiana dell'interno, oltre all'esemplare citato da Leoni (1907).

I reperti sinora noti (Napoli, Roma, Salerno, Isole) sembravano indicare che Phaeopterus unicolor potesse essere una specie termofila a distribuzione costiera, anche se un esame più attento dei dati (Cerchio, Bocognano, Ucciani, Aritzo) potrebbe suggerire una preferenza altitudinale. La scarsità dei reperti non ci permette di trarre conclusioni ma sembra piuttosto indicare che la specie sia legata a querceti e castagneti di media quota. Phaeopterus avrebbe quindi una distribuzione simile, seppure certamente più termofila, a quella del genere Omalisus.

La biologia rimane sconosciuta e la femmina ignota, anche se si suppone possa essere non molto dissimile da quella di Omalisus fontisbellaquaei Geoffroy, 1785: cioè brachittera e legata alla lettiera. È possibile che Phaeopterus unicolor sia una specie molto sensibile all'azione antropica sui boschi, soffrendo sia il disboscamento che l'inquinamento ambientale.
Come per Omalisus, i ritrovamenti appaiono legati ad una certa casualità, ma le dimensioni più ridotte e il colore scuro rendono questa specie ancora più sfuggente. Benché alcuni autori abbiano segnalato catture su piante basse (Champion, 1911; Sainte-Claire Deville, 1921), la battitura dei rami ("ombrellatura") o le trappole ad intercettazione del volo potrebbero essere metodi di cattura proficui. Ricerche specializzate condotte in boschi di latifoglie, non antropizzati, di località collinari ben esposte potrebbero incrementare i dati su questa specie e fornire ulteriori informazioni per chiarirne la biologia.

\section{Ringraziamenti}

Ringraziamo il Prof. Roberto A. Pantaleoni (Università di Sassari), studioso delle opere di Achille Costa, per l'aiuto bibliografico e i preziosi suggerimenti riguardo all'anno di descrizione della specie, e Fernando Angelini (Francavilla Fontana), per le informazioni sul proprio reperto.

\section{BIBLIOGRAFIA}

Anonimo, 1857 - Memorie approvate nell'anno 1856. Scienze Naturali. Memorie della Reale Accademia delle Scienze di Napoli, 2: XXXII-XXXIII.

Bargagli P., 1873 - Materiali per la fauna entomologica dell'Isola di Sardegna. Coleotteri (Contin.). Bollettino della Società Entomologica Italiana, 5 (1): 3449.

Baudi di Selve F., 1872 - Europæ et circummediterraneæ Faunæ Dascillidum et Malacodermatum specierum, quæ Comes Dejean in suo Catalogo ed. $3^{\text {a }}$ consignavit, ex ejusdem collectione in R. Taurinensi Musæo asservata, cum auctorum hodierne recepta denominatione, collatio. Berliner Entomologische Zeitschrift, 15: 89130.

Bertolini S., 1872-1878 - Catalogo sinonimico e topografico dei Coleotteri d'Italia. Tipografia Cenniniana, Firenze.

Bertolini S., 1904 - Catalogo dei Coleotteri d'Italia. Rivista Italiana Scienze naturali, Siena.

Bocak L. \& Brlik M., 2008 - Revision of the family Omalisidae (Coleoptera, Elateroidea). Insect Systematics \& Evolution, 39 (2): 189-212.

Bourgeois J., 1882a - Séance du 14 Juin $1882,1^{\mathrm{e}}$ note. Annales de la Société Entomologique de France, Bulletin des Séances (VI), 2: C-CI.

Bourgeois J., 1882b - Monographie des Lycides de l'ancien-monde. L'Abeille: Journal d'Entomologie, 20: $1-120$.

Bourgeois J., 1884-1892 - Cébrionides, Dascillides, Malacodermes. In: Faune Gallo-Rhénane ou species des insectes qui habitent la France, la Belgique, la Hollande, le Luxembourg, la Prusse Rhénane, le Nassau et le Valais; Coléoptères. Revue d'Entomologie, Caen, 4.

Champion G. C., 1911 - A trip to Sardinia in 1910. The Entomologist's Monthly Magazine (II), 22: 219223. 
Costa A., 1856 - De quibusdam novis insectorum generibus, ossia caratteri di nuovi generi e specie in quella descritte, spettanti tutti alla Fauna Napoletana (Sunto della memoria). Rendiconto della Società Reale Borbonica - Accademia delle Scienze (Nuova Serie), 5: 17-20.

Costa A., 1857 - De quibusdam novis insectorum generibus descriptis, iconibusque illustratis. Memorie della Reale Accademia delle Scienze di Napoli, 2: 219-233.

Haliday A. H., [1869] - [Letters from Alexander Henry Haliday to Hermann Loew]. Lucca. <http:// en.wikisource.org/wiki/Letters from Alexander Henry_Haliday_to_Hermann_Loew_July_24,_1863and_undated $>$ (conservate nella biblioteca della Royal Entomological Society di Londra).

Heyden L. v., Reitter E. \& Weise J., 1891 - Catalogus Coleopterorum Europæ, Caucasi et Armeniæ rossicæ. R. Friedländer \& Sohn, Berlin; E. Reitter, Mödling; Revue d'Entomologie, Caen.

Heyden L. v., Reitter E., Weise J., 1906 - Catalogus Coleopterorum Europæ, Caucasi et Armeniæ Rossicæ, Ed. 2. R. Friedländer \& Sohn, Berlin; E. Reitter, Paskau; Revue d'Entomologie, Caen.

Hicker R., 1925 - Catalogus Coleopterorum regionis palaearcticæ: Diversicornia. Malacodermata. A. Winkler, Wien, 5: 487-578.

Kleine R., 1942 - Bestimmungstabelle der Lycidae. Bestimmungs-Tabellen der europäischen Coleopteren. E. Reitter, Troppau, 123.
Leoni G., 1907 - Specie e varietà nuove o poco cognite ed appunti biologici sopra i coleotteri italiani ( $2^{\mathrm{a}}$ nota). Rivista Coleotterologica Italiana, 5: 183-199.

Luigioni P., 1929 - I Coleotteri d'Italia. Catalogo sinonimico-topografico-bibliografico. Memorie Pontificia Accademia delle Scienze Nuovi Lincei (II), 13: 1-1160.

Pic M., 1938 - Notes diverses, nouveautés (Suite). L'Échange, Revue Linnéenne, 54 (474): 13-14.

Porta A., 1929 - Fauna Coleopterorum Italica: Diversicornia. Stabilimento Tipografico Piacentino, Piacenza, 3.

Reitter E., 1911 - Fauna Germanica - Die Käfer des Deutschen Reiches nach der analytischen Methode bearbeitet. K. G. Lutz, Stuttgart, 3.

Sainte-Claire Deville J., 1914 - Catalogue critique des Coléoptères de la Corse. Revue d'Entomologie, Caen.

Sainte-Claire Deville J., 1921 - Catalogue critique des Coléoptères de la Corse ( $2^{\mathrm{e}}$ supplément). Annales de la Société Entomologique de France, 89: 377-404.

Sainte-Claire Deville J., 1926 - Catalogue critique des Coléoptères de la Corse ( $3^{\mathrm{e}}$ supplément). Annales de la Société Entomologique de France, 95: 113-130.

Schaufuss C. F. C., 1916 - Calwer's Käferbuch: Einführung in die Kenntnis der Käfer Europas. Sechste Auflage. E. Schweizerbart'sche Verlagsbuchhandlung, Stuttgart, 1.

Winkler J. R., 1989 - The families Omalisidae emend. n. and Lycidae (Coleoptera) in Slovak National Museum, Bratislava. Annotationes zoologicae et botanicae 189: 1-18. 